AN. MED. INTERNA (Madrid) Vol. 18, N. $^{\circ} 12$, pp. $650-654,200$

\title{
Los principios bioéticos: ¿se aplican en la situación de enfermedad terminal?
}

\author{
A. AZULAY TAPIERO
}

Servicio de Medicina Interna. Unidad de Cuidados Paliativos. Hospital Dr. Moliner. Valencia

\author{
BIOMEDICAL ETHICS PRINCIPLES: ARE THEY APPLIED IN \\ TERMINAL ILLNESS SITUATION?
}

\begin{abstract}
RESUMEN
La aplicación de los principios bioéticos fundamentales (beneficencia, no-maleficencia, autonomía y justicia) garantizaría una asistencia adecuada al enfermo que está en situación de enfermedad terminal. Lamentablemente, en nuestro ámbito, esto no se hace de forma sistemática.

Para garantizar una asistencia de calidad a este grupo de enfermos es necesario que los profesionales sanitarios que se enfrentan con la enfermedad terminal reciban una adecuada formación en cuestiones de bioética, que se aplique una metodología correcta para resolver los conflictos y que se impliquen las instituciones del estado para que las unidades de Cuidados Paliativos cubran la asistencia de toda la población subsidiaria. De lo contrario, se produce un atentado contra los principios bioéticos fundamentales, en especial contra un principio de primer nivel, el principio de justicia.
\end{abstract}

PALABRAS CLAVE: Bioética. Enfermedad terminal. Cuidados Paliativos.

Azulay Tapiero A. Los principios bioéticos: ¿se aplican en la situación de enfermedad terminal? An Med Interna (Madrid) 2001; 18: 650-654.

\begin{abstract}
Comprehensive practice of biomedical ethics principles (beneficence, non-maleficence, autonomy and justice) would guarantee appropriate assistance to patients with terminal diseases. Unfortunately today, these principles aren't put into practice in a systematic way.

In order to guarantee quality care to this particular group of patients, it is necessary that all health care professionals dealing with terminal illness receive a comprehensive education in bioethics matters and that an appropriate methodology is followed in order to resolve subsequent conflicts. Furthermore, public institutions should be involved so that Palliative Care Units provide assistance to the entire population rather than select cases. If we can't provide comprehensive care, we are working against the basic bioethics principles, especially the fundamental principle of justice.
\end{abstract}

KEY WORDS: Bioethics. Terminal illness. Palliative Care.
El personal sanitario, cuando se enfrenta con la enfermedad progresiva e incurable que progresa irremediablemente hacia la muerte, cuando no es posible la curación, es invadido por un estado de duda constante sobre qué es lo más adecuado hacer para beneficiar al enfermo. La actitud que debe tomar ante los dilemas que aparecen no debe ser producto de la intuición ni de su estado de ánimo en ese momento, sino que debe estar basada en una reflexión prudente, informada, discutida y compartida por el resto de los miembros del equipo interdisciplinar y, a ser posible, conociendo y teniendo muy en cuenta cuáles son las preferencias del enfermo.

Un método utilizado para intentar resolver estos conflictos éticos en la labor diaria con el enfermo es el propuesto hace ya más de 20 años por Beauchamp y Childress.

Antes de responder a la pregunta que se plantea en el título de este trabajo, es necesario conocer, aunque sea de forma muy elemental, qué es la bioética, cuáles son sus objetivos y comentar la "teoría de los cuatro principios" desarrollada por los autores anteriormente citados.

\section{CONCEPTO Y OBJETIVOS DE LA BIOÉTICA}

La ética es la parte de la Filosofía que estudia la bondad o la malicia intrínseca de los actos y de las conductas humanas.

La ética debe apoyarse en la racionalidad y en el terreno filosófico, y no en la religión, el derecho o los códigos deontológicos. Lo ético puede no ser legal (por ejemplo, la eutanasia es considerada una actuación éticamente correcta para determinadas personas, pero es ilegal en casi todos los países) y lo legal puede no ser ético (por ejemplo, la pena de muerte es una acción contraria a la ética para amplios sectores sociales, pero está legalmente constituida en varios países). La deontología y la ley no deben ser un instrumento adecuado para definir lo conveniente o lo correcto ante un caso concreto, no se debe "judicializar" la vida de las personas, a no ser que se quiera conseguir una forma de totalitarismo (1), aunque sí será necesario legislar unos mínimos éticos aceptados por todos, los principios éticos legales (2), y exigibles a todos por igual (3). Ninguna ideología ni ninguna creencia religiosa deben imperar en una

Trabajo aceptado: 3 de Septiembre de 2001

Correspondencia: Armando Azulay Tapiero. C/ Espinosa nº 1, pta. 1 - 46008 Valencia. e-mail: azulay-arm@gva.es 
sociedad plural y secularizada, donde deben tener cabida todas las corrientes ideológicas, con la condición de que respeten la dignidad de las personas y los derechos humanos.

La Medicina, como es fácil de suponer, desde los tiempos más remotos, desde su inicio, ha estado relacionada con la ética, ya que su objetivo, por encima de cualquier otro, siempre ha sido conseguir el bien del ser humano enfermo.

El término "bioética" procede del griego: "bios" (vida) y "ethos" (comportamiento, costumbre). El objetivo principal de la bioética es por tanto el estudio de la relación existente entre la vida y los principios o pautas de la conducta humana.

La palabra "bioética" es un neologismo que aparece por primera vez en el año 1971, en el libro del oncólogo norteamericano Van Potter titulado Bioethics: a Bridge to the Future ("Bioética: un puente al futuro") (4); este autor la define como "la disciplina que combina el conocimiento biológico con el de los valores humanos". Según la obra coordinada por Warren Reich, Encyclopedia of Bioethics, la bioética se define como "el estudio sistemático de la conducta humana en el área de las ciencias de la vida y del cuidado sanitario, en cuanto se examina esta conducta a la luz de los valores y principios morales" (5).

En la práctica, el objetivo de la bioética, en lo que respecta a la Medicina, será la búsqueda de soluciones concretas a casos clínicos concretos, siempre que se originen conflictos entre valores. La bioética entrará en acción cuando aparezca la posibilidad de elegir entre dos o más opciones, que a veces podrán ser completamente contradictorias. El dilema consiste en buscar la forma más adecuada para tomar las decisiones correctas, es decir, cómo elegir entre lo correcto y lo incorrecto, cómo diferenciar lo que es bueno de lo que es malo. Cuando entran en juego los problemas esenciales de la vida y la muerte, la responsabilidad aumenta y esta búsqueda se hace más complicada. Para encontrar la solución al dilema que se plantea se debe actuar con libertad, pero no de forma empírica o intuitiva, sino con prudencia, con un método adecuado y en un abordaje interdisciplinario, ya que todas las ramas del saber que tienen por objeto al ser humano tienen elementos que aportar en la búsqueda de soluciones, de ahí deriva la importancia de la constitución de "comités de bioética" en los centros hospitalarios, una de cuyas misiones será la de orientar y ayudar a los profesionales sanitarios en la solución de estos problemas.

La ética médica, a lo largo de toda su historia, siempre consideró que el personal sanitario sólo estaba obligado a conseguir el máximo beneficio del enfermo y que no tenía sentido preocuparse por otros motivos. En las últimas décadas, el juicio moral de un acto médico ha variado; entre sus objetivos, además de conseguir el máximo beneficio del enfermo, se debe tener en cuenta otras dimensiones o aspectos:

-Las preferencias del paciente: puede que "lo mejor" para nosotros, personal sanitario, no coincida con "lo mejor" para el paciente, y sería ilícito imponer nuestra propia idea del bien a otras personas.

— La calidad de vida subjetiva.

- Los factores sociales y económicos.

-El bien de la sociedad en su conjunto.

\section{PRINCIPIOS BIOÉTICOS}

Para resolver el problema de los conflictos que aparecen en el momento de tomar las decisiones en el área biomédica, surge la bioética de orientación principalista basada en "Los Cuatro Principios" formulados por Beauchamp y Childress. La bioética principalista consiste en conseguir un método sistemático de reflexión que permita elegir una solución correcta ante un dilema bioético.

En el año 1979, estos dos autores norteamericanos, basándose en el "Informe Belmont", que fue encargado en el año 1974 por el Congreso Norteamericano para elaborar una guía acerca de los procedimientos éticos que debían regir la investigación con seres humanos y dado a conocer en el año 1978, publicaron un libro titulado Principles of Biomedical Ethics ("Principios de Ética Biomédica") (6).

El contenido de esta obra se divide en tres partes fundamentales:

-Estudio de las principales corrientes éticas, dando importancia a los elementos más constructivos de las mismas.

—Propuesta de unos llamados "principios", considerados como una pauta o guía útil en la práctica diaria para la toma de decisiones ante casos complejos que puedan dar lugar a discrepancias. Constituye la parte central de la obra.

-Las llamadas "virtudes" que debería poseer el profesional sanitario para una atención correcta, integral y humanizada del individuo enfermo, que, a diferencia de los principios, no serían obligaciones éticas. Se refieren, entre otras, a la compasión, a la capacidad de discernir sobre qué es lo relevante en cada circunstancia, a la integridad, a la empatía, a la prudencia en el momento de tomar las decisiones, etc.

Los principios bioéticos que propusieron eran: beneficencia, no-maleficencia, autonomía y justicia. El principio bioético más elemental y sobre el que se basan los enumerados anteriormente es el "respeto a la dignidad de la persona", basado en un enfoque integral y humanista de la asistencia sanitaria.

Principio de Beneficencia: manda hacer el bien. Es el principio más evidente de todos ya que el personal sanitario ha sido educado y formado para hacer el bien, no sólo al individuo enfermo, sino a la sociedad en su conjunto. Se basa en que los procedimientos diagnósticos y terapéuticos que se apliquen deben beneficiar al paciente, es decir, ser seguros y efectivos.

Principio de no-maleficencia: se basa en el principio hipocrático de Primum non nocere, es decir, "ante todo, no hacer daño". También es un principio muy evidente porque ningún profesional sanitario deberá utilizar sus conocimientos o su situación para ocasionar perjuicios al enfermo. En la práctica se refiere a que el balance entre los beneficios y los riesgos de cualquier actuación médica debe ser siempre a favor de los beneficios.

Principio de autonomía: se refiere a la capacidad que tiene el enfermo para decidir, siempre que exprese su deseo. Al contrario que los anteriores, es un principio que siempre ha estado ausente de la tradición médica, a pesar de la gran importancia que ha adquirido en los últimos años; durante muchos siglos el paciente nunca ha participado en la toma de decisiones y el médico era quien decidía; el deber del médico era "hacer el bien" al paciente, y el de éste, aceptarlo (7). El enfermo, por el mero hecho de serlo, carecía de capacidad para elegir.

Este principio es sumamente importante porque:

-Obliga a informar al enfermo, si así lo desea, sobre el diagnóstico, pronóstico y posibilidades terapéuticas, con sus riesgos y beneficios.

-Permite al enfermo rechazar todo tipo de tratamiento o elegir uno distinto al propuesto.

-Debería permitir al enfermo, dentro de lo posible y con 
las limitaciones legales vigentes, elegir el momento, lugar y forma de su muerte.

Para promover este principio se ha propugnando el llamado "consentimiento informado" como forma de garantizar que el paciente ha recibido una información adecuada sobre el acto médico a aplicar y que manifiesta su acuerdo. El consentimiento informado es tanto más importante cuanto mayores sean los riesgos del tratamiento propuesto o de la técnica de exploración a la que se someta y cuantas más posibilidades existan de fracaso. El "Manual de Ética" del Colegio de Médicos Americanos lo define de la siguiente manera (8): "El consentimiento informado consiste en la explicación a un paciente atento y mentalmente competente de la naturaleza de su enfermedad, así como el balance entre los efectos de la misma y los riesgos y beneficios de los procedimientos terapéuticos recomendados, para a continuación solicitarle su aprobación para ser sometido a estos procedimientos. La presentación de la información al paciente debe ser comprensible y no sesgada; la colaboración del paciente debe ser conseguida sin coerción; el médico no debe sacar partido de su potencial dominio psicológico sobre el paciente". Esta definición, aunque contiene todos los elementos necesarios para la correcta aplicación del consentimiento informado, tiene un pequeño fallo, es incompleta, ya que donde cita a los procedimientos terapéuticos, debería añadir también a las técnicas de diagnóstico (9).

Principio de Justicia: este principio se basa en dos hechos:

- Todas las personas, por el mero hecho de serlo, tienen la misma dignidad, independientemente de cualquier circunstancia, y por tanto, son merecedoras de igual consideración y respeto.

- Hay que luchar por una distribución justa y equitativa de los siempre limitados recursos sanitarios para conseguir el máximo beneficio en la comunidad, evitando desigualdades en la asistencia sanitaria.

Este principio convierte al personal sanitario en gestor y administrador de los recursos y de los servicios, que deberá utilizar de una forma efectiva y eficiente, evitando actuaciones sanitarias inadecuadas.

Estos cuatro principios adquirieron rápidamente una gran importancia, ya que resultaron muy útiles para los profesionales sanitarios que no eran expertos en cuestiones de bioética y que actuaban generalmente de forma empírica o intuitiva ante cualquier dilema moral que aparecía en su labor diaria con el enfermo.

Durante muchos siglos, los principios éticos más relevantes en la actuación sanitaria han sido los de beneficencia y nomaleficencia, dando lugar a un paternalismo autoritario, bien intencionado y aceptado por la sociedad. En las últimas décadas, los principios de autonomía y de justicia cada vez adquieren mayor relevancia, sobre todo en los países anglosajones.

Entre estos cuatro principios los autores no establecieron ningún orden jerárquico, insistiendo en que todos tienen la misma fuerza y son de aplicación obligatoria, aunque no absolutos, ya que hay excepciones. Cada caso concreto permitirá la negociación, es decir, sólo las circunstancias podrán establecer un orden jerárquico entre ellos. Un ejemplo que aparece con determinada frecuencia en la práctica de los cuidados paliativos sería el siguiente: atendiendo al principio de no-maleficencia, ocasionar la muerte es algo que en principio se considera siempre moralmente incorrecto y por tanto contrario a la ética médica, pero en determinadas circunstancias, administrar fármacos que puedan adelantar la muerte ("principio del doble efecto") puede tenerse en cuenta cuando interviene la autonomía del paciente en una situación de sufrimiento muy intenso.
Otro aspecto a tener en cuenta es que según qué marco de referencia o doctrina ética se practique, podrá variar la interpretación de estos principios generales de la bioética. Un ejemplo típico sería el siguiente: en una doctrina puramente utilitarista, la opción del aborto como derecho de la mujer embarazada a decidir sobre todo lo que acontece en su cuerpo (principio de autonomía) será válido y por tanto aceptado. En un marco ético personalista que defienda la defensa de la vida desde la fecundación, el derecho a la vida del ser humano aún no nacido es lo fundamental, por lo que se relega la autonomía de la madre a un plano inferior, anteponiendo el principio de no-maleficencia a favor del ser humano que aún no ha nacido.

Según Diego Gracia (10), sí existe un orden jerárquico entre estos principios. Diferencia dos niveles:

- Primer nivel o nivel universal: son los que no se refieren a la relación entre el personal sanitario y el paciente y son independientes de la voluntad de éste. Obligan a todos los ciudadanos. Constituyen lo que se llama "ética de mínimos" y deben ser garantizados por el estado. El gobierno que no pueda garantizar estos dos principios se encontraría "bajo mínimos morales". Estos principios son:

- Principio de no-maleficencia: la Medicina como ciencia inocua, que no persigue el hacer daño. Es un principio más general y obligatorio que el de Beneficencia porque habrá ocasiones en que un médico no esté obligado a tratar a un enfermo, pero sí lo estará siempre a no ocasionarle ningún daño, o dicho de otra manera, si una persona no quiere, no podemos imponerle el bien; por el contrario, si una persona quiere que le hagamos daño, no se lo podemos hacer por más que nos lo pida.

- Principio de justicia: la Medicina debe tratar a todos sin ningún tipo de discriminación, debiendo proteger más a los más desfavorecidos. El bien común o el bien de todos debe ser siempre superior al bien de individuos concretos. Cuando no se cumple con este principio se produce maleficencia

-Segundo nivel o nivel particular: son los principios que se refieren a la relación existente entre el personal sanitario y el paciente. Constituyen la llamada "ética de máximos" porque obligan a la persona y no a los demás; la persona, en el caso que nos ocupa, el enfermo, tiene libertad para elegir su aplicación o no.

- Principio de beneficencia: pretende que la actuación del sistema sanitario sea beneficiosa para el paciente. La interpretación de este principio es relativa y depende de la voluntad de la persona que recibe esta actuación supuestamente benéfica y de que la acepte como tal beneficio: nadie puede beneficiar a otro contra su voluntad. Por todo esto, beneficencia y autonomía son principios inseparables y siempre tendrá más valor el segundo.

- Principio de Autonomía: tiene en cuenta la libertad y la responsabilidad del paciente que, bien informado, decide lo que es bueno para él.

Cuanto más aguda es la enfermedad y más eficaz es su tratamiento, menos interviene en la práctica el principio de autonomía y más el de beneficencia, y cuanto más crónica es la enfermedad o más cuestionado esté su tratamiento, como ocurre en la situación de enfermedad terminal, más veces se reclama la autonomía.

En líneas generales, los grandes problemas surgen cuando se plantean conflictos entre los principios de un mismo nivel. El conflicto más frecuente es el que surge entre los principios de Autonomía y Beneficencia, al rechazar el paciente o su representante legal un determinado tratamiento o prueba diagnóstica. Cuando surge un conflicto entre prin- 
cipios de distinto nivel, en principio, deberá prevalecer el del nivel superior.

Mención especial merece la práctica médica en el SIDA (11) en donde aparece un hecho diferencial que da lugar a un importante dilema bioético: el secreto profesional versus la protección de la pareja del paciente infectado, es decir, ¿debe notificarse la enfermedad a la pareja del paciente sin dar éste su consentimiento?; desde el punto de vista ético, la prevención de la enfermedad debe prevalecer sobre el principio de autonomía y la intimidad del paciente

\section{LOS PRINCIPIOS BIOÉTICOS EN EL ENFERMO EN SITUACIÓN DE ENFERMEDAD TERMINAL}

En la práctica diaria, cuando se atiende a un enfermo en situación de enfermedad terminal, lo habitual es la ausencia de conflictos entre estos cuatro principios bioéticos y el enfermo solicitará que se favorezca la aplicación de todos ellos para que se atiendan todas las necesidades que plantea:

-Necesita que, aunque no exista un tratamiento con finalidad curativa, se intente dar una solución integral a sus problemas, tanto físicos como psicoemocionales, sociales y espirituales (Principio de Beneficencia).

-Necesita que no se le dañe de forma innecesaria utilizando procedimientos terapéuticos o diagnósticos que se hayan mostrado inútiles o fútiles o que sean considerados desproporcionados (principio de no-maleficencia).

-Necesita que se le trate con confianza, respetando su intimidad y su autonomía, que se le informe si así lo desea y que se tenga en cuenta su opinión en el momento de decidir qué es lo mejor para él (principio de autonomía).

-Necesita que, si existe un sistema de salud universal, como ocurre en nuestro ámbito, cubra sus necesidades, independientemente de cualquier circunstancia, y que no se le discrimine por ningún motivo (principio de justicia).

El personal sanitario que quiera conseguir una correcta atención a este tipo de enfermos debe ir encaminado a dar solución a cada una de estas demandas.

Lamentablemente, hay varias circunstancias que orientan a que muchas veces no se aplican estos cuatro principios en su totalidad, en especial los principios de autonomía y justicia.

La supuesta beneficencia de un acto médico ante la enfermedad sin posibilidad de curación que progresa de forma irremediable a la muerte es muy relativa ya que la frontera existente entre perjuicio y beneficio queda muy difuminada y muchas veces dependerá del propio criterio del enfermo, de su concepción de la vida y de su escala de valores personales, aspectos que el personal asistencial debe tener muy en cuenta (12); por ejemplo, ¿beneficia o perjudica la colocación de una sonda nasogástrica para nutrir a un enfermo que está en situación de enfermedad terminal?, no es posible dar una respuesta a esta pregunta si no se conoce la opinión del enfermo (de ahí la estrecha relación que hay entre los principios de beneficencia y autonomía) y si no se estudia el caso concreto detenidamente y desde un punto de vista interdisciplinar. Sin embargo, ante una enfermedad con tratamiento curativo, este dilema no se suele plantear, o se plantea con mucha menor intensidad, ya que está mucho más clara la frontera existente entre lo que beneficia y lo que perjudica al paciente; volviendo al ejemplo de la sonda nasogástrica, su colocación para nutrir e hidratar durante unos días a un enfermo que ha sufrido un traumatismo maxilofacial y que ha sido sometido por ello a una intervención quirúrgica y en cuyo postoperatorio está indicado el reposo absoluto de la actividad masticatoria y deglutoria, no cabe ninguna duda de que siempre le beneficiará, aunque también aquí podría haber conflicto con el principio de autonomía, en el caso de que el paciente, por el motivo que sea, se negase a su colocación.

Debido a los grandes avances técnicos y farmacológicos ocurridos en las últimas décadas, que a veces incluso permiten posponer el momento de la muerte, actitud que nunca se debe adoptar ante la situación de enfermedad terminal, se produce otro hecho que se da con más frecuencia de la deseada, que consiste en la práctica de medidas compatibles con la obstinación terapéutica, también llamada ensañamiento o encarnizamiento terapéutico, en un intento irracional de luchar contra la muerte próxima e inevitable. Esta práctica atenta contra el principio de no-maleficencia ya que muchas veces, lo que se hace al adoptar estas medidas, es dañar al enfermo de una forma innecesaria, aplicando tratamientos que ocasionan sufrimiento.

El principio de autonomía en la cultura latina es ampliamente ignorado cuando nos encontramos con un enfermo con el diagnóstico de enfermedad terminal. Hay una tendencia por parte de la familia, muchas veces apoyada por el personal sanitario que lo atiende y su tradicional actitud paternalista y a veces autoritaria, a ocultar al enfermo información sobre su enfermedad y su pronóstico, y sin información es imposible poder decidir y participar en la toma de decisiones. Aún en el caso de que se informe al enfermo, ¿es la información que se da aséptica y neutra? o, por el contrario ¿es sesgada y parcial con el fin de ocultar el mal pronóstico o para manipular la posible decisión del paciente?. El "consentimiento informado", aunque sólo sea de forma verbal, debería estar presente ante cualquier decisión terapéutica o diagnóstica en un enfermo en situación de enfermedad terminal, y no sólo cuando participa en un ensayo clínico, donde si se cumplimenta, es porque la ley así lo exige.

Con respecto al principio de justicia conviene puntualizar que, si bien deben existir ciertos límites para la creación de determinados servicios médicos financiados por un sistema sanitario público, como podrían ser, a modo de ejemplo, los que se dedican a aplicar técnicas de fecundación in vitro o algunas intervenciones de cirugía estética, la medicina paliativa no debe entrar en este grupo de servicios y debe ser considerada como un servicio sanitario básico y necesario, nunca accesorio, al que se tenga derecho en una sanidad pública y universal como la nuestra.

El principio de justicia no se aplica en su totalidad debido a que, en nuestro ámbito, los programas de Cuidados Paliativos, que hoy por hoy constituyen la mejor y más eficaz forma de atención a estos enfermos, sólo prestan su cobertura en determinadas áreas sanitarias y a un pequeño porcentaje de la población que podría beneficiarse de ellos, tal y como queda reflejado en el último Directorio publicado por la Sociedad Española de Cuidados Paliativos (SECPAL), en el año 2000 (13). No toda la población se puede beneficiar de estos programas a pesar de ser necesidades básicas para un gran número de personas y no requerir su implantación enormes recursos económicos. ¿Qué ocurriría si por ejemplo no hubiese atención neurológica o cardiovascular que cubriese a toda la población?: sin lugar a dudas se produciría un gran debate social y político que solucionaría el problema de manera inmediata; ¿por qué no ocurre lo mismo ante la falta manifiesta de unidades especializadas en cuidados paliativos y más si tenemos en cuenta que un gran número de personas, cuando mueren, no lo hacen de forma súbita, sino tras un perio- 
do más o menos largo de sufrimiento?, quizá la respuesta esté en la negación que toda la sociedad, incluida la clase política, hace de la muerte y todo lo que le rodea y en la poca capacidad reivindicativa que tiene el enfermo aquejado de una enfermedad progresiva e incurable que, una vez fallecido, no podrá protestar o reclamar por la atención (o desatención) que ha recibido. Existe discriminación en este terreno: los cuidados paliativos no están disponibles para todas aquellas personas que los necesitan; dicho de otra manera, la calidad de la atención prestada a un enfermo que está en situación terminal dependerá de la existencia de una unidad de cuidados paliativos que cubra su área de salud, y esto es un atentado contra el principio de justicia.

La deficiencia de unidades de cuidados paliativos que garanticen, en la medida de lo posible, un adecuado control del sufrimiento favorece el desarrollo de movimientos sociales que están a favor de la eutanasia, práctica que origina un debate social cada vez más intenso y que se realiza en varias partes del mundo, a pesar de ser, al menos hoy en día, ilegal en la mayoría de los países, pero que cuenta con un gran número de adeptos dentro de la ciudadanía en general y del personal sanitario en particular (14), quizá debido a que, en la conciencia colectiva, siempre se asocia a la muerte con el sufrimiento.

La Organización Mundial de la Salud estableció que "los gobiernos deben asegurar que se ha dedicado especial atención a las necesidades de sus ciudadanos en el alivio del dolor y los cuidados paliativos antes de legislar sobre la eutanasia" (15). Como señala Jaime Sanz, "con una cobertura del $100 \%$ de los cuidados paliativos no será necesario introducir nuevas figuras legales (suicidio asistido y/o eutanasia activa voluntaria)" (16), pero también hay que decir que, por muy bien que funcionen las unidades de cuidados paliativos, siempre existirá el sufrimiento ante la enfermedad terminal y no siempre se podrá evitar del todo la demanda de eutanasia por parte de aquellos pacientes que viven una situación insoportable y piden ayuda para poner fin a dicho sufrimiento (17). Los cuidados paliativos no resolverán todos los casos de petición de eutanasia, pero sí una gran parte de ellos.

\section{CONCLUSIÓN}

La Medicina Paliativa, por su gran componente humanitario y por su defensa al respeto a la vida y a la dignidad de la persona, constituye la forma de asistencia más eficaz para los pacientes que se encuentran en situación de enfermedad terminal, ya que la filosofía en la que se basa garantiza, entre otras cosas, una aplicación estricta y sistemática de los principios bioéticos fundamentales.

La Medicina Paliativa, por definición, debe procurar mejorar la calidad de vida del enfermo, debe luchar por conseguir el mayor bienestar posible, su objetivo debe ser evitar los problemas físicos, psicológicos, sociales y espirituales o, si esto no es posible, procurar que el enfermo se adapte a ellos en las mejores condiciones posibles, debe evitar actuaciones innecesarias que provoquen sufrimiento y también debe fomentar la autonomía del enfermo para hacerle partícipe en la toma de unas decisiones que son muy importantes para él, para lo cual es indispensable una adecuada información y comunicación. Con ello, los principios de beneficencia, nomaleficencia y autonomía quedan garantizados.

Pero esto no es suficiente, no se puede construir un sistema moral coherente y completo en las ciencias biomédicas si solamente aplicamos estos tres principios. Esto se consigue cuando se añade el principio de justicia (18).

El principio de justicia, el que se refiere a la distribución equitativa de los recursos sanitarios y a que hay que tratar a todas las personas por igual evitando cualquier tipo de discriminación, queda hoy en día, en lo que se refiere a los cuidados paliativos, relegado a un segundo plano, quizá porque la formación ética del gestor sanitario, salvo excepciones, que sin duda las hay, es escasa y, posiblemente, en la atención al enfermo en situación de enfermedad terminal, valore más el ahorro económico a corto y medio plazo que la rentabilidad social.

Desde una unidad de cuidados paliativos, en lo que respecta al principio de justicia, sólo se podrá actuar en el ámbito de la decisión clínica, en el día a día con el paciente, pero esto sigue sin ser suficiente. Las Instituciones del Estado deberán, si se quiere cumplir con este principio en su totalidad, planificar, desarrollar y gestionar de forma eficiente las políticas sanitarias para lograr la máxima cobertura posible y así evitar discriminaciones en este campo, que hoy por hoy se siguen dando. Como se ha expuesto anteriormente, citando de nuevo a Diego Gracia, el gobierno que no es capaz de garantizar un principio ético de primer nivel o nivel universal, como es el principio de justicia, se encuentra "bajo mínimos morales".

\section{Bibliografía}

1. Simón Lorda $\mathrm{P}$, Barrio Cantalejo M. Un marco histórico para una nueva disciplina. Med Clin (Barc) 1995; 105: 583-97.

2. Martín Escudero JC. Consentimiento y confidencialidad en la práctica médica del SIDA. An Med Interna (Madrid) 2001; 18: 287-8.

3. Cortina A. Ética mínima. Madrid: Tecnos, 1986.

4. Potter R Van. Bioethics: A Bridge to the Future. Englewood Cliffs (N.J.) 1971.

5. Reich WT. Encyclopedia of Bioethics. Introducción. I. New York, Free Press-McMillan Publishing Co. Inc. 1978; 19.

7. Sanz Ortiz J. Bioética y Medicina Paliativa. En: Gómez Sancho M. Medicina Paliativa en la Cultutra Latina. Madrid: Aran Ed. S.A., 1999: 1161-76.

8. Ad hoc Committee on Medical Ethics, American College of Physicians. American College of Physicians Manual. Ann Intern Med 1984; 101: 129-137.

9. Simón Lorda P, Concheiro Carro L. El consentimiento informado: teoría y práctica (I). Med Clin (Barc) 1993; 100: 659-63.
10. Gracia D. Procedimientos de Decisión en Ética Clínica. Madrid, Ed. Eudema S.A., 1991: 124-34.

11. Monés Xiol J, Barrio Medrano JL. Aspectos éticos de la práctica médica en el SIDA. An Med Interna (Madrid) 2000; 17: 171-3.

12. Azulay A. Dilemas bioéticos en la situación de enfermedad terminal y en el proceso de la muerte. Med Pal 2000; 7: 145-56.

13. Centeno C, Hernánsanz S, Flores LA, López-LaraF. Directorio de Cuidados Paliativos. España: Ed. SECPAL, 2000.

14. Martínez Urionabarrenetxea K. Eutanasia y Cuidados Paliativos: ¿Amistades Peligrosas?. Med Clin (Barc) 2001; 116: 142-5.

15. World Health Organization. Cancer Pain Relief. Ginebra: WHO, 1990.

16. Sanz Ortiz J. Final de la Vida: ¿Puede ser confortable? Med Clin (Barc) 2001; 116: 186-90.

17. Ferrer E. La Eutanasia como excepción. JANO 2000; 59 (1357): 27-8.

18. Gracia D. Fundamentos de Bioética. Madrid. Ed. Eudema S.A., 1989; 202. 